Commun. Korean Math. Soc. 27 (2012), No. 2, pp. 243-255

http://dx.doi.org/10.4134/CKMS.2012.27.2.243

\title{
ON AN INVERSE PROBLEMS FOR LAPLACE EQUATIONS WITH POTENTIAL TERMS ON ELECTRICAL NETWORKS
}

\author{
Ji Chan Chung, Du Hyeong Kim, and Tae Hoon Kwon
}

\begin{abstract}
In this paper, we deal with an inverse problem for electrical resistor networks to detect the location of nodes where an extraordinary currents flow into or out of the nodes proportional to the potentials on them. To achieve the goal, we solve a special type of mixed boundary value problem for Laplace equations with potential terms on rectangular networks which plays a role as a forward problem. Then we solve an inverse problem to develop an algorithm to locate the node where the extraordinary current flows on it at most four times of measurements of potential and current on its boundary.
\end{abstract}

\section{Introduction}

A network is a structure consisted by the set of nodes and the set of links which connects selected nodes and has been widely used as a tool for modeling physical, biological and social phenomena. As technologies develop, electrical networks tend to be more miniaturized or structurally complicated so that when we have some problem on a part of the network, it is almost impossible to investigate the whole network. For this reason, developing a method to recover the whole network using partial data becomes more and more important for practical applications.

Suppose an electrical network which has nodes where the extraordinary currents flow into or out of the nodes proportional to the potentials on them. This phenomenon is mathematically modeled as an equation which is said to be the discrete Laplace equation with potential term or discrete Schrödinger equation and various type of boundary value problems for the equation have been studied so far (see, for example, [1]). The main consideration of this paper is to develop an efficient algorithm to detect the location of the erroneous nodes using the electrical potentials and currents measured on its boundary. There have been numerous works (see, for example, $[3,4,5]$ ) on locating the edges whose conductivities are perturbed, which is called the faulty resistor problem, but little has been so far studied about locating the nodes on which external

Received July 20, 2010.

2010 Mathematics Subject Classification. Primary 42A15; Secondary 15B05.

Key words and phrases. trigonometric series, Hankel determinant, signal processing. 
currents flows. From the economical viewpoint, it is no less important to develop an algorithm with minimal measurements than to develop an accurate algorithm. With this in mind, in this paper, we provide an algorithm which needs at most four times of measurements.

This research has been studied under the following two restrictions. First, every electrical networks in this study are assumed to be the resistor networks without inductors or capacitors, although studying the RLC circuits are very important for practical purpose. We leave it for the future works. Second, at the stage of studying inverse problems and developing algorithm, we only deal with a special type of networks - the rectangular networks. It is also leaved to the future work to develop algorithms which can be applied to more general type of networks. But in authors' opinion, rectangular network is not an excessive restriction when one applies our algorithm to solve inverse problems on continuum by using the method of discretizing domain such as finite difference method (see, for example, [2]).

\section{Preliminaries}

In this section, we recall notations and basic results on the theory of electrical networks. For more details, it is recommended to refer to $[1,3,4,5]$. Graph consists a finite set $V$ of nodes and a set $E$ of links which connects two selected nodes. A graph $G(V, E)$ is said to be simple if it has neither multiple links nor loops. If $x$ and $y \in V$ are connected by a link, it is denoted as $x \sim y$. Representing the link as $\{x, y\}$, it is natural to regard the set $E$ of links as the subset $\{\{x, y\} \mid x, y \in V x \sim y\}$ of the set $V \times V$. A weighted graph $G(V, E ; \omega)$ is a graph $G(V, E)$ associated with a weight function $\omega: V \times V \rightarrow[0, \infty)$ satisfying

$$
\left\{\begin{array}{l}
\omega(x, y)=0 \quad \Leftrightarrow \quad x \sim y \\
\omega(x, y)=\omega(y, x), \quad x, y \in V .
\end{array}\right.
$$

From now on, the weighted graph is said to be the network. Since the set of edge $E$ is uniquely determined by the weight function, the network $G(V, E ; \omega)$ is simplified as $G(V ; \omega)$. A network $G(V ; \omega)$ is said to be connected if for each pair of nodes $x$ and $y$, there exists a sequence of vertices $\left\{x_{1}, \ldots, x_{n}\right\}$ such that $x=x_{1} \sim x_{2} \sim \cdots \sim x_{n}=y$. Throughout this paper, every networks are regarded as connected networks, unless otherwise noted.

The networks we consider in this paper are electrical resistor networks (ERNs). For two nodes $x \sim y$ of an $\operatorname{ERN} G(V ; \omega)$, the weight $\omega(x, y)$ plays a role of the reciprocal of its resistance $R(x, y)$ on the link connecting $x$ and $y$, namely, $\omega(x, y)=1 / R(x, y), x \sim y$. Note that for each $x$ and $y \in V$ with $x \sim y$, the weight $\omega(x, y)$ represents the conductivity between the nodes. If a network satisfy Kirchhoff's law on each of its node, then it can be mathematically modeled by

$$
\Delta_{\omega} u(x)=0, \quad x \in V
$$


where $\Delta_{\omega} u(x):=\sum_{y \in V}[u(y)-u(x)] \omega(x, y)$. Here, the function $u: V \rightarrow \mathbf{R}$ represents the potential on the nodes. If a network $G(V ; \omega)$ has $N$ nodes so that $V$ is represented by $V=\left\{x_{1}, x_{2}, \ldots, x_{N}\right\}$, then it is easy to see that (1) can be rewritten as the following system of linear equation:

$$
\left(\begin{array}{cccc}
-d_{\omega} x_{1} & \omega\left(x_{1}, x_{2}\right) & \cdots & \omega\left(x_{1}, x_{N}\right) \\
\omega\left(x_{2}, x_{1}\right) & -d_{\omega} x_{2} & \cdots & \omega\left(x_{2}, x_{N}\right) \\
\vdots & \vdots & \ddots & \vdots \\
\omega\left(x_{N}, x_{1}\right) & \omega\left(x_{N}, x_{2}\right) & \cdots & -d_{\omega} x_{N}
\end{array}\right)\left(\begin{array}{c}
u\left(x_{1}\right) \\
u\left(x_{2}\right) \\
\vdots \\
u\left(x_{N}\right)
\end{array}\right)=\left(\begin{array}{c}
0 \\
0 \\
\vdots \\
0
\end{array}\right)
$$

where $d_{\omega} x:=\sum_{y \in V} \omega(x, y)$. Therefore, the square matrix in the above equation is said to be the Kirchhoff matrix. Throughout this paper, the Kirchhoff matrix is denoted as $\Delta_{\omega}$. We sometimes partition the set of nodes $V$ into two disjoint subsets $S$ and $\partial S$ so that $V=S \cup \partial S$. The subsets $S$ and $\partial S$ of $V$ are said to be the interior and the boundary of $V$, respectively. In what follows, we use the notation $\bar{S}$, instead of $V$, to denote the set of nodes if it is partitioned into the interior $S$ and the boundary $\partial S \neq \emptyset$ and $z_{1} \nsim z_{2}$ for each $z_{1}$ and $z_{2} \in \partial S$. The normal derivative of a function $u: \bar{S} \rightarrow \mathbf{R}$ at each boundary node is defined as

$$
\frac{\partial u}{\partial n}(z)=\sum_{y \in S}[u(z)-u(y)] \omega(y, z) .
$$

Note that $\frac{\partial u}{\partial n}(z)=-\Delta_{\omega} u(z), z \in \partial S$. For an $\operatorname{ERN} G(\bar{S} ; \omega)$, if the potentials are given arbitrary on its boundary nodes, then the potentials on interior nodes are determined by Kirchhoff's law. The unique determinacy of the potentials on interior is guaranteed by the following well known result (see, for example, $[3])$.

Theorem 2.1. For an $\operatorname{ERN} G(\bar{S} ; \omega)$, there exists a unique solution $u: \bar{S} \rightarrow \mathbf{R}$ of the following Dirichlet boundary value problem (DBVP)

$$
\begin{cases}-\Delta_{\omega} u(x)=0, & x \in S \\ u(z)=\sigma(z), & x \in \partial S\end{cases}
$$

\section{Boundary value problems for Laplace equation with potential terms on networks}

From now on, we consider an electrical network where an extraordinary current flows on each node in $S$ proportional to the potential on it. If a potential $\sigma: \partial S \rightarrow \mathbf{R}$ is imposed on its boundary, then this phenomenon can be mathematically modeled by the following DBVP for Laplace equation with potential terms on networks.

$$
\begin{cases}-\Delta_{\omega} u(x)+q(x) u(x)=0, & x \in S \\ u(z)=\sigma(z), & z \in \partial S,\end{cases}
$$

where the potential term $q(x) u(x)$ means the extraordinary current flows on the node $x$. The main concern in this subsection is to prove the unique solvability of the above DBVP (2) (Theorem 3.1). We also consider Neumann and mixed 
boundary value problems which deal with the case that currents and mixtures of currents and potentials are imposed on the boundary, respectively (Theorems $3.2 \sim 3.3)$. Let $\left\{x_{1}, \ldots, x_{K}\right\}$ and $\left\{x_{K+1}, \ldots, x_{N}\right\}$ denote the set $S$ and the set $\partial S$, respectively. Note that $(2)$ can be represented by the following system of linear equation

$$
\left(-\Delta_{\omega}+D_{q}\right) U=\Psi
$$

where $D_{q}$ denotes the diagonal matrix

$$
\begin{gathered}
\left(\begin{array}{cccccc}
q\left(x_{1}\right) & \cdots & 0 & 0 & \cdots & 0 \\
\vdots & \ddots & \vdots & \vdots & \ddots & \vdots \\
0 & \cdots & q\left(x_{K}\right) & 0 & \cdots & 0 \\
0 & \cdots & 0 & 0 & \cdots & 0 \\
\vdots & \ddots & \vdots & \vdots & \ddots & \vdots \\
0 & \cdots & 0 & 0 & \cdots & 0
\end{array}\right), \\
U:=\left(\begin{array}{c}
u\left(x_{1}\right) \\
\vdots \\
u\left(x_{K}\right) \\
\sigma\left(x_{K+1}\right) \\
\vdots \\
\sigma\left(x_{N}\right)
\end{array}\right) \text { and } \quad \Psi:=\left(\begin{array}{c}
0 \\
\vdots \\
0 \\
\psi\left(x_{K+1}\right) \\
\vdots \\
\psi\left(x_{N}\right)
\end{array}\right) .
\end{gathered}
$$

We divide the matrix $-\Delta_{\omega}+D_{q}$ by four parts as

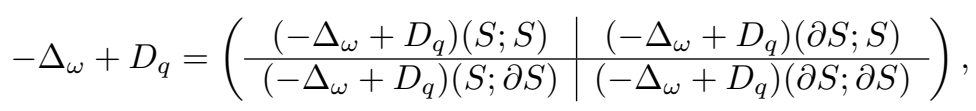

where

$$
\left(-\Delta_{\omega}+D_{q}\right)(S ; S)=\left(\begin{array}{ccc}
d_{\omega} x_{1}+q\left(x_{1}\right) & \cdots & -\omega\left(x_{1}, x_{K}\right) \\
\vdots & \ddots & \vdots \\
-\omega\left(x_{K}, x_{1}\right) & \cdots & d_{\omega} x_{K}+q\left(x_{K}\right)
\end{array}\right)
$$

Since it is well known that the submatrix

$$
-\Delta_{\omega}(S ; S):=\left(\begin{array}{cccc}
d_{\omega} x_{1} & -\omega\left(x_{1}, x_{2}\right) & \cdots & -\omega\left(x_{1}, x_{K}\right) \\
-\omega\left(x_{2}, x_{1}\right) & d_{\omega} x_{2} & \cdots & -\omega\left(x_{2}, x_{K}\right) \\
\vdots & \vdots & \ddots & \vdots \\
-\omega\left(x_{K}, x_{1}\right) & -\omega\left(x_{K}, x_{2}\right) & \cdots & d_{\omega} x_{K}
\end{array}\right)
$$

of $-\Delta_{\omega}$ is positive definite, it is easy to see that if $q(x) \geq 0, x \in S$, then the submatrix $\left(-\Delta_{\omega}+D_{q}\right)(S ; S)$ is positive definite. On the other hand, the known fact that $-\Delta_{\omega}$ is nonnegative definite guarantees that for a function $q \neq 0$ with $q(x) \geq 0, x \in S$, the matrix $-\Delta_{\omega}+D_{q}$ is positive definite. 
We state below the various boundary value problems for the Laplace equation with potential terms. Since they are easily proved by using the above facts, we omit their proofs.

Theorem 3.1. For an $\operatorname{ERN} G(\bar{S} ; \omega)$ and a function $q: S \rightarrow \mathbf{R}$ with $q(x) \geq$ $0, x \in S$, there exists a unique solution $u: \bar{S} \rightarrow \mathbf{R}$ of the following Dirichlet boundary value problem $(D B V P)$

$$
\begin{cases}-\Delta_{\omega} u(x)+q(x) u(x)=0, & x \in S \\ u(z)=\sigma(z), & x \in \partial S\end{cases}
$$

Moreover, the solution $u$ is represented as

$$
\left[u_{S}\right]=\left[\left(-\Delta_{\omega}+D_{q}\right)(S ; S)\right]^{-1}\left[\left(\Delta_{\omega}-D_{q}\right)(S ; \partial S)\right][\sigma],
$$

where $\left[u_{S}\right]:=\left(u\left(x_{1}\right), \ldots, u\left(x_{K}\right)\right)^{T}$ and $\left.[\sigma]:=\left(\sigma\left(x_{K+1}\right), \ldots, \sigma\left(x_{N}\right)\right)\right)^{T}$.

Theorem 3.2. For an $E R N G(\bar{S} ; \omega)$ and a nonzero function $q: S \rightarrow \mathbf{R}$ with $q(x) \geq 0, x \in S$, there exists a unique solution $u: \bar{S} \rightarrow \mathbf{R}$ of the following Neumann boundary value problem (NBVP)

$$
\begin{cases}-\Delta_{\omega} u(x)+q(x) u(x)=0, & x \in S \\ \frac{\partial u}{\partial n}(z)=\psi(z), & x \in \partial S .\end{cases}
$$

Moreover, the solution $u$ is represented as

$$
[u]=\left[\left(-\Delta_{\omega}+D_{q}\right)(S ; S)\right]^{-1}[0 \mid \psi],
$$

where $[u]:=\left(u\left(x_{1}\right), \ldots, u\left(x_{N}\right)\right)^{T}$ and $\left.[0 \mid \psi]:=\left(0, \ldots, 0, \psi\left(x_{K+1}\right), \ldots, \psi\left(x_{N}\right)\right)\right)^{T}$.

Theorem 3.3. For an $E R N G(\bar{S} ; \omega)$ with boundary $\partial S=\partial S_{1} \cup \partial S_{2}\left(\partial S_{1} \cap\right.$ $\left.\partial S_{2}=\emptyset\right)$ and a function $q: S \rightarrow \mathbf{R}$ with $q(x) \geq 0, x \in S$, there exists a unique solution $u: \bar{S} \rightarrow \mathbf{R}$ of the following Dirichlet boundary value problem (DBVP)

$$
\begin{cases}-\Delta_{\omega} u(x)+q(x) u(x)=0, & x \in S \\ \frac{\partial u}{\partial n}(z)=\psi(z), & z \in \partial S_{1} \\ u(z)=\sigma(z), & x \in \partial S_{2}\end{cases}
$$

Moreover, by indexing the boundary nodes as $\partial S_{1}=\left\{x_{K+1}, \ldots, x_{K+m}\right\}$ and $\partial S_{2}=\left\{x_{K+m+1}, \ldots, x_{N}\right\}$, the solution $u$ is represented as

$$
\begin{aligned}
{\left[u_{S \cup \partial S_{1}}\right]=} & {\left[\left(-\Delta_{\omega}+D_{q}\right)\left(S \cup \partial S_{1} ; S \cup \partial S_{1}\right)\right]^{-1} } \\
& \times\left\{[0 \mid \psi]+\left[\left(\Delta_{\omega}-D_{q}\right)\left(S \cup \partial S_{1} ; \partial S_{2}\right)\right][\sigma]\right\},
\end{aligned}
$$

where $\left[u_{S \cup \partial S_{1}}\right]:=\left(u\left(x_{1}\right), \ldots, u\left(x_{K+m}\right)^{T},[0 \mid \psi]:=\left(0, \ldots, 0, x_{K+1}, \ldots, x_{K+m}\right)\right.$ and $\left.[\sigma]:=\left(\sigma\left(x_{K+m+1}\right), \ldots, \sigma\left(x_{N}\right)\right)\right)^{T}$. 


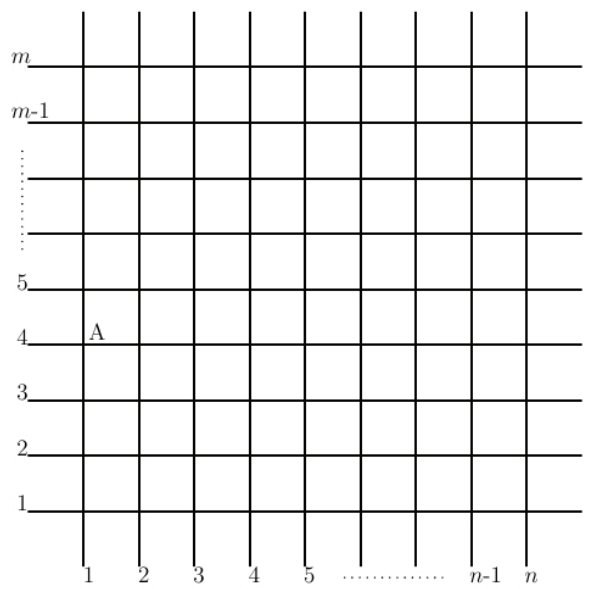

Figure 1.

\section{An inverse problem on rectangular networks}

An $m \times n$ rectangular network $G(\bar{S} ; \omega)$ is a network which is constructed as follows. The set of nodes $\bar{S}$ is the set of the lattice points $(i, j)$ for which $i=0,1,2, \ldots, n+1$ and $j=0,1,2, \ldots, m+1$ with the four corner points $(0,0),(0, M+1),(N+1,0)$ and $(N+1, N+1)$ are excluded. The interior $S$ of $\bar{S}$ is the set of nodes $(i, j), 1 \leq i \leq N, 1 \leq j \leq M$. The set of edges $E$ is the set of horizontal and vertical line segment which connects each pair of adjacent nodes $x$ and $y \in S$ or which connects each boundary node $z \in \partial S$ to its adjacent interior node $x \in S$. See Figure 1 .

Let $G(\bar{S} ; \omega)$ be the rectangular network which satisfy

$$
-\Delta_{\omega} u(x)+q(x) u(x)=0, \quad x \in S .
$$

The main consideration of this subsection is to locate the support of the function $q(x)$ using a mixture of Dirichlet and Neumann boundary condition on its boundary nodes. Especially, we are interested in the case that $q(x)$ is supported at one node $x_{0} \in S$, namely, $q(x)=k \delta_{x_{0}}, x \in S$ for some $k \in \mathbf{R} \backslash\{0\}$. Here $\delta_{a}$ denotes the Kroneker delta centered at $a$.

\subsection{A forward problem}

In what follows, our interest will be restricted to $N \times N$ rectangular networks. We use the notation $\partial S_{N}, \partial S_{E}, \partial S_{W}$ and $\partial S_{S}$ to partition the boundary into four parts as $\partial S_{N}=\{(i, N+1) \mid i=1,2, \ldots, N\}, \partial S_{W}=\{(0, i) \mid i=$ $1,2, \ldots, N\}, \partial S_{S}=\{(i, 0) \mid i=1,2, \ldots, N\}$ and $\partial S_{E}=\{(N+1, i) \mid i=$ $1,2, \ldots, N\}$. 


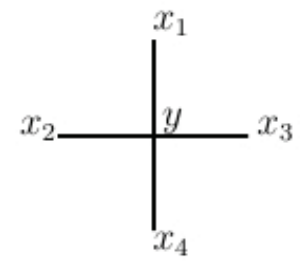

Figure 2.

Lemma 4.1. For the network given as Figure 2, we have the following.

(i) If $\Delta_{\omega} u(y) \geq 0($ resp. $\leq 0)$ and $u(y) \geq u\left(x_{i}\right)$ (resp. $\left.\leq u\left(x_{i}\right)\right), i=1,2,3$, then $u\left(x_{4}\right) \geq u(y)$ (resp. $\left.\leq u(y)\right)$. Moreover, if we append the assumption that $u(y)>u\left(x_{i}\right)$ (resp. $\left.<u\left(x_{i}\right)\right)$ for some $i=1,2,3$, then $u\left(x_{4}\right)>u(y)$ (resp. $<u(y))$.

(ii) If $\Delta_{\omega} u(y)>0($ resp. $<0)($ resp. $=0)$ and $u(y)=u\left(x_{i}\right)=0, i=1,2,3$, then $u\left(x_{4}\right)>0($ resp. $<0)($ resp. $=0)$.

Proof. They can be easily proved from the following fact:

$$
u\left(x_{4}\right)=\frac{\Delta_{\omega} u(y)+u(y) d_{\omega} y-\sum_{i=1}^{3} u\left(x_{i}\right) \omega\left(x_{i}, y\right)}{\omega\left(x_{4}, y\right)} .
$$

To solve the main result - the inverse problem to locate the support of $q(x)$, we first need to solve a certain type of MBVP, which play a role of the forward problem.

Theorem 4.2. Let $\psi: \partial S_{E} \rightarrow \mathbf{R}$ be given. The solution $u: \bar{S} \rightarrow \mathbf{R}$ of the following $M B V P$

$$
\begin{cases}-\Delta_{\omega} u(x)+q(x) u(x)=0, & x \in S \\ u(z)=\frac{\partial u}{\partial n}(z)=0, & z \in \partial S_{N} \cup \partial S_{W} \\ \frac{\partial u}{\partial n}(z)=\psi(z), & z \in \partial S_{E}\end{cases}
$$

exists and is uniquely determined.

Proof. From the above boundary condition, we have $u(x)=0$ for each interior node $x$ adjoint to some nodes in $\partial S_{N} \cup \partial S_{W}$, precisely, $x=(1, i)$ or $x=i, N$, $i=1,2, \ldots, N$. Then it follows from the result in (ii) of Lemma 4.1, it is easy to see that $u(x)=0$ in $\partial S_{N} \cup \partial S_{W} \cup T$ where

$$
T=\{x=(i, j) \mid 1 \leq i \leq N, i \leq j \leq N\} .
$$

See Figure 3 for the case $N=9$. Next we calculate the potential $u(x)$ uniquely at the nodes $x \in \bar{S} \backslash\left[\partial S_{N} \cup \partial S_{W} \cup T\right]$ following the numerical order as in Figure 4 (see also Figure 5 for the case $N=9$ ). This completes the proof. 


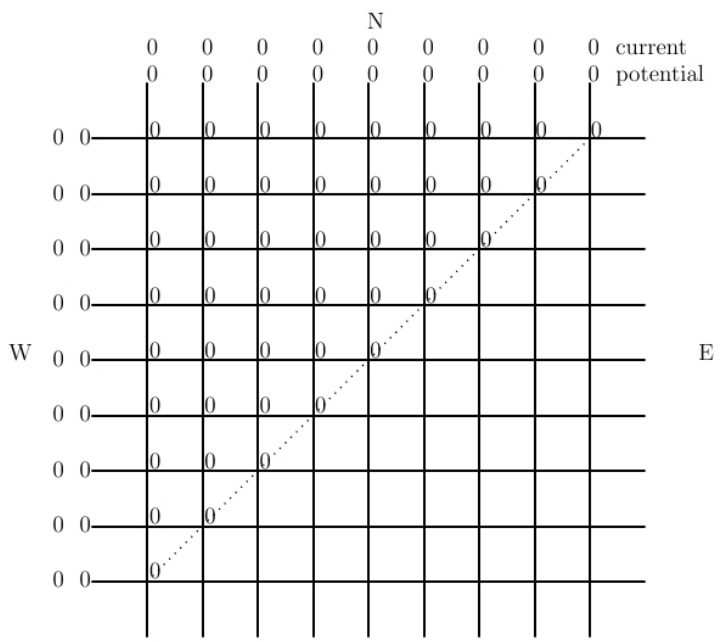

Figure 3 .

Remark 4.3. According to the proof of the above theorem, we have

$$
u \equiv 0, \quad \text { in } T
$$

and moreover, if the current $\psi: \partial S_{E} \rightarrow \mathbf{R}$ is defined by

$$
\psi(z)= \begin{cases}c, & z=(N+1, N) \\ 0, & z \in \partial S_{E} \backslash\{(N+1, N)\}\end{cases}
$$

for some $c \in \mathbf{R}$, then it follows from Lemma 4.1 that

$$
u(z) \neq 0, \quad x \in \bar{S} \backslash T
$$

by calculating the potentials in the numerical order given in Figure 4.

\subsection{Exact localization of the support of the potential term}

Consider an $N \times N$ network which satisfy Kirchhoff's law in all interion nodes except at $x_{0} \in S$, where an extraordinary current flows proportional to the potential on it. If one impose a certain type of boundary condition applying the potential $u(z)=0$ and the current $\frac{\partial u}{\partial n}(z)=0$ for $z \in \partial S_{N} \cup \partial S_{W}$ and the current $\frac{\partial u}{\partial n}(z)=\psi(z)$ for $z \in \partial S_{E}, \psi$ is defined the same as in (4), then this phenomenon is expressed as the MBVP (3), where the potential term $q: S \rightarrow \mathbf{R}$ is defined by $q(x)=k \delta_{x_{0}}(x), x \in S$ for some $k \in \mathbf{R} \backslash\{0\}$. The main interest in this subsection is to provide an algorithm to locate the $x_{0} \in S$ using the boundary observation $\sigma(z)=u(z)$ on $\partial S_{S} \cup \partial S_{E}$. Note that Theorem 4.2 guarantees the unique existence of the potential $\sigma: \partial S_{S} \cup \partial S_{E} \rightarrow \mathbf{R}$. 


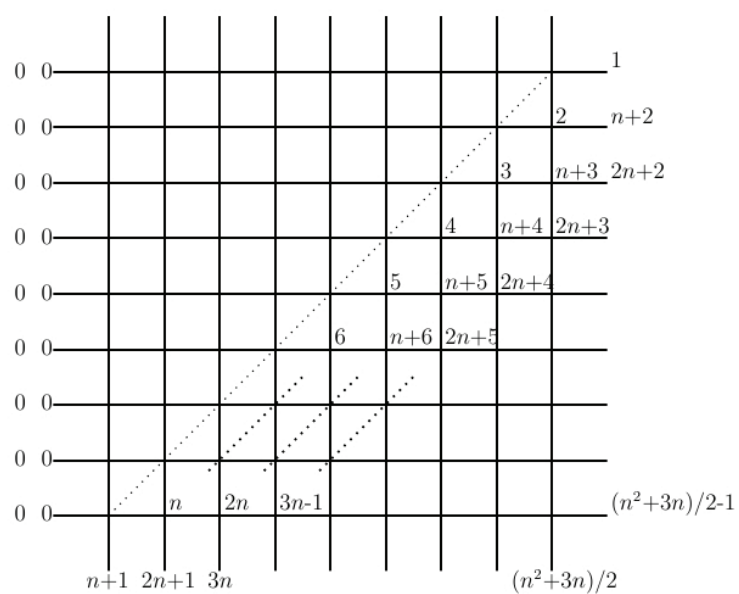

Figure 4 .

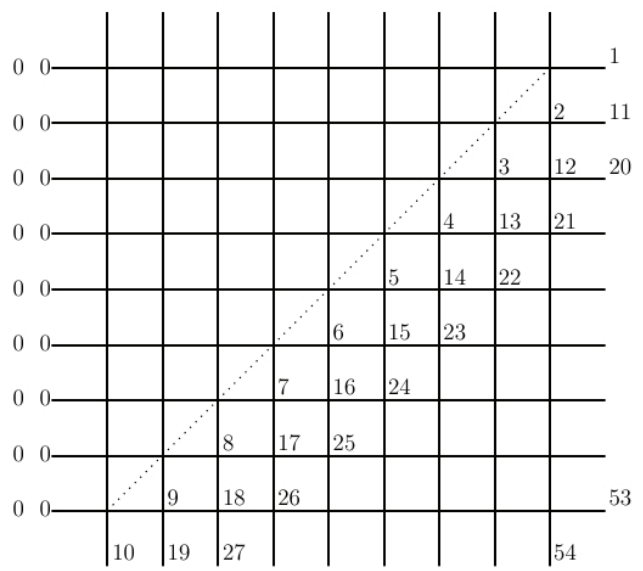

Figure 5 .

Let the node $(1,0) \in \partial S_{S}$ be named as $z_{1}$ and other nodes in $\partial S_{S} \cup \partial S_{E}$ as $z_{2}, z_{3}, \ldots, z_{2 N}$ with clockwise increasing index numbers, precisely, $z_{2}=$ $(2,0), \ldots, z_{N}=(N, 0), z_{N+1}=(N+1,1), \ldots, z_{2 N}=(N+1, N)$. See Figure 6 . 


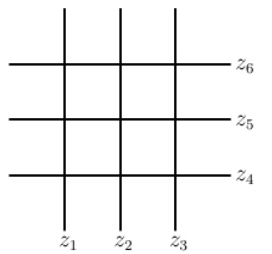

Figure 6.

We state and prove the main result of this paper.

Theorem 4.4. For given $\psi: \partial S_{E} \rightarrow \mathbf{R}$ defined the same as in (4) and $x_{0} \in S$, let $u: \bar{S} \rightarrow \mathbf{R}$ be the solution of the equation (3) and $u_{0}: \bar{S} \rightarrow \mathbf{R}$ be the background potential, i.e., the solution of the equation

$$
\begin{cases}-\Delta_{\omega} u_{0}(x)=0, & x \in S \\ u_{0}(z)=\frac{\partial u_{0}}{\partial n}(z)=0, & z \in \partial S_{N} \cup \partial S_{W} \\ \frac{\partial u_{0}}{\partial n}(z)=\psi(z), & z \in \partial S_{E} .\end{cases}
$$

(i) If $x_{0} \in T=\{(i, j) \in S \mid i \leq j\}$, then $u \equiv u_{0}$ on $\partial S_{S} \cup \partial S_{E}$.

(ii) If $x_{0}=\left(i_{0}, j_{0}\right) \in S \backslash T$, then we have

$$
u(z) \neq u_{0}(z), \quad z \in A_{x_{0}} \subset \partial S_{S} \cup \partial S_{E}
$$

and

$$
u(z)=u\left(z_{0}\right), \quad z \in \partial S_{S} \cup \partial S_{E} \backslash A_{x_{0}},
$$

where

$$
A_{x_{0}}=\left\{z_{i_{0}-j_{0}+n} \in \partial S_{S} \cup \partial S_{E} \mid n=1,2, \ldots, 2 j_{0}-1\right\}
$$

(see Figure 7 and Figure 8).

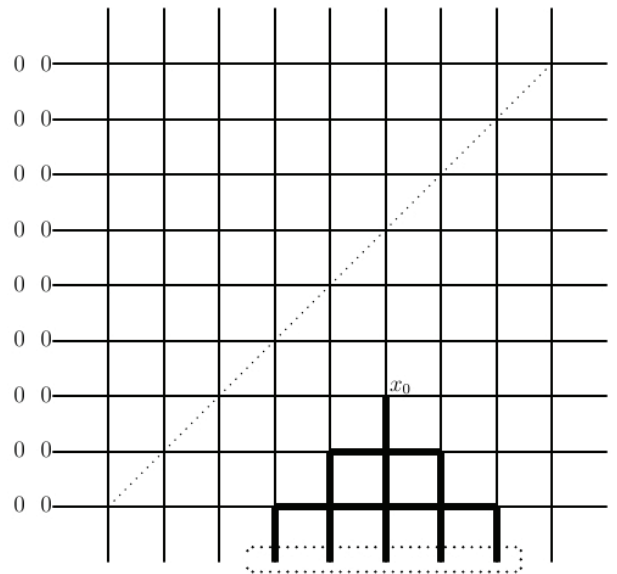

Figure 7 . 


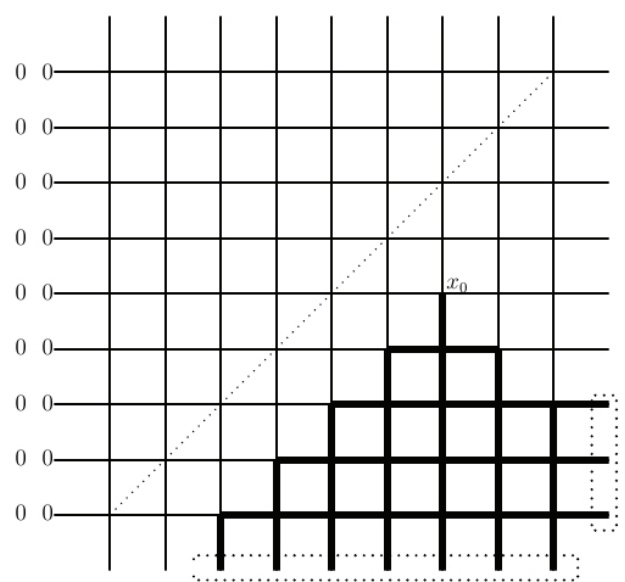

Figure 8.

Proof. Note that $U: \bar{S} \rightarrow \mathbf{R}$ defined by $U(z)=u(z)-u_{0}(z), z \in \bar{S}$ is the solution of the following mixed boundary value probelm

$$
\begin{cases}-\Delta_{\omega} U(x)+q(x) u(x)=0, & x \in S \\ U(z)=\frac{\partial U}{\partial n}(z)=0, & \partial S_{N} \cup \partial S_{W} \\ \frac{\partial U}{\partial n}(z)=0, & z \in \partial S_{E} .\end{cases}
$$

If $x_{0} \in T$, then it follows from Remark 4.3 that $u\left(x_{0}\right)=0$ and hence we have

$$
q(x) u(x)=k \delta_{x_{0}}(x) u(x)=0, \quad x \in S .
$$

Calculating $U(x), x \in \bar{S} \backslash T$ in numerical order given in Figure 4, it follows from Lemma 4.1 that $U(x)=0, x \in \bar{S} \backslash T$, which proves (i). On the other hand, if $x_{0} \in \bar{S} \backslash T$, then again by Remark 4.3, we have $q\left(x_{0}\right) u\left(x_{0}\right) \neq 0$ and calculating $U(x), x \in \bar{S} \backslash T$ the same way as we have used to prove (i), we can easily show that $U(x) \neq 0, x \in A_{x_{0}}$, which proves (ii).

\section{Algorithm.}

For given $\psi: \partial S_{E} \rightarrow \mathbf{R}$ with $\psi(N+1, N) \neq 0$ and $x_{0} \in S$, let $u: \bar{S} \rightarrow \mathbf{R}$ be the solution of the MBVP (3) where

$$
q(x)=k \delta_{x_{0}}(x) \quad \text { for some } k \in \mathbf{R} \backslash\{0\}
$$

and

$$
U(z):=u(z)-u_{0}(z), \quad z \in \partial S_{S} \cup \partial S_{E},
$$

where $u_{0}$ is the background potential satisfying (5). Based on Theorem 4.4, we provide an algorithm to detect the position $x_{0}$ of the source term. Like the preceding, the boundary nodes in $\partial S_{S} \cup \partial S_{E}$ is named by $z_{1}, \ldots, z_{2 N}$, starting from the node $z_{1}=(1,0)$ with clockwise increasing index numbers, ending with $z_{2 N}=(N+1, N)$.

Step I. Observe $U(z), z \in \partial S_{S} \cup \partial S_{E}$. It follows from Theorem 4.4 that either (i) $U(z) \neq 0$ for $z=z_{i}, z_{i+1}, \ldots, z_{i+2 n}$ for some $i$ and $n$ or (ii) $U(z) \equiv 0$ 
on $\partial S_{S} \cup \partial S_{E}$. In the case (i), we conclude that $x_{0}=(i+n, n+1)$ and complete the algorithm. In the case (ii), we conclude that $x_{0} \in T$ and move to Step II.

Step II. Rotate the network upside down and repeat Step I again (See Figure $9)$. In the case (i), we again conclude that $x_{0}=(i+n, n+1)$ and complete the algorithm. In the case (ii), we now conclude that $x_{0}$ lies in the diagonal nodes (with negative slope), i.e., $x_{0} \in\{(i,-i+N+1) \mid i=1,2, \ldots, N\}$ and move to Step III.

Step III. Rotate the network clockwise through 90 degrees and repeat Step I again (See Figure 10). In the case (i), we again conclude that $x_{0}=(i+n, n+1)$ and complete the algorithm. In the case (ii), we now conclude that $x_{0} \in$ $\left\{(i, j) \mid i=1,2, \ldots,\left[\frac{N}{2}\right]-1\right\}$ and move to Step $I V$.

Step IV. Repeat Step III again. In the case (i), we again conclude that $x_{0}=(i+n, n+1)$ and complete the algorithm. In the case (ii), we conclude that $x_{0}=\left(\left[\frac{N}{2}\right]+1,\left[\frac{N}{2}\right]+1\right)$ and complete the algorithm.
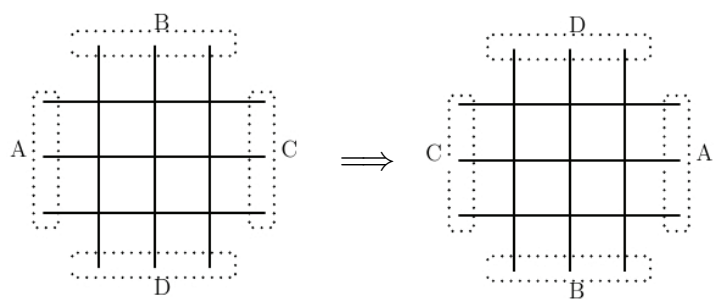

Figure 9.
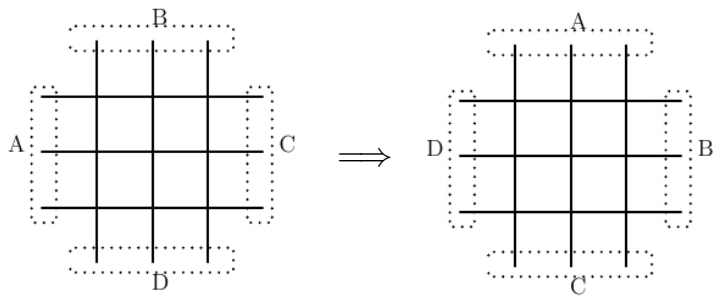

Figure 10.

Remark 4.5. The case (ii) in the Step $I V$ only occurs when $N$ is an odd number.

\section{References}

[1] E. Bendito, A. Carmona, and A. Encinas, Potential theory for Schrödinger operators on finite networks, Rev. Mat. Iberoamericana 21 (2005), no. 3, 771-818.

[2] L. Borcea, V. Druskin, and F. Guevara Vasquez, Electrical impedance tomography with resistor networks, Inverse Problems 24 (2008), no. 3, 035013, 31 pp.

[3] S.-Y. Chung and C. A. Berenstein $\omega$-Harmonic functions and inverse conductivity problems on networks, SIAM J. Appl. Math. 65 (2005), no. 4, 1200-1226.

[4] E. B. Curtis and J. A. Morrow, The Dirichlet to Neumann map for a resistor network, SIAM J. Appl. Math. 51 (1991), no. 4, 1011-1029.

[5] Y. Mun, The faulty resistor problems and the inverse source problems for rectangular electrical networks, Commun. Korean Math. Soc. 24 (2009), no. 3, 467-479. 
Ji Chan Chung

Department of Electrical Engineering

KAIST

DAEJEON 305-701, KOREA

E-mail address: jcchung93@hanmail.net

Du HyeOng KiM

Gyeongai Science High School

SuwON 440-800, KoreA

E-mail address: du1204@naver.com

TAE HoOn KWON

Gyeongai Science High School

SuWON 440-800, KoreA

E-mail address: thk0317@naver.com 